\title{
Synergistic pH effect for reversible shuttling aptamer-based biosensors between graphene oxide and target molecules
}

\author{
Po-Jung Jimmy Huang, ${ }^{a}$ Ravindra Kempaiah ${ }^{a}$ and Juewen Liu*a
}

\author{
Received 19th April 2011, Accepted 11th May 2011 \\ DOI: $10.1039 / \mathbf{c 1 j m 1 1 7 0 2 \mathrm { e }}$
}

DNA aptamers are known to desorb from graphene oxide (GO) surface in the presence of target molecules. We demonstrate herein that the binding equilibrium can be shifted by simply tuning the solution $\mathrm{pH}$. At lower $\mathrm{pH}$, the aptamer/GO binding is enhanced while aptamer/target binding is weakened, making this system a regenerable biosensor without covalent conjugation.

Understanding and controlling binding between biomolecules and inorganic materials is important in basic surface science research, biomedical engineering, biosensor development and nanotechnology. ${ }^{1-4}$ An interesting recent example is the adsorption of non-structured single-stranded DNAs on graphene oxide (GO) and the subsequent desorption upon forming double-stranded or well-folded structures. Graphene and GO are newly discoered materials with many useful mechanical, electric, and optical properties. ${ }^{5-8}$ For example, GO can effectively quench adsorbed fluorophores. These properties have been used to make highly sensitive fluorescent biosensors for metal ions, ${ }^{9-11}$ small molecules, ${ }^{12-16}$ proteins,,${ }^{17-}$ 20 and DNA. ${ }^{10,18,21-26}$ For example, mixing a fluorescently labeled aptamer with GO resulted in quenched fluorescence. Upon addition of the target molecule, the aptamer can bind to the target and desorb from the surface, resulting in fluorescence enhancement (Figure 1, step 1). After detection, however, it is difficult to re-adsorb the DNA and wash away the target molecule since there is no covalent linkage between the aptamer and the surface. If a re-adsorption mechanism can be introduced, this system can serve as a regenerable sensor. Herein, we report that the GO/aptamer system can be reversibly operated by changing the solution $\mathrm{pH}$. We demonstrate the concept for both adenosine aptamer and a $\mathrm{Hg}^{2+}$ binding DNA.

Since most aptamers are selected at close to the neutral $\mathrm{pH}$, we hypothesize that lowering the $\mathrm{pH}$ might have an adverse effect on aptamer binding. To test this, a biophysical technique known as isothermal titration calorimetry (ITC) was employed, where the heat generated by mixing the target and aptamer was measured. The unmodified adenosine aptamer $(60 \mu \mathrm{M})$ was warmed up to $90{ }^{\circ} \mathrm{C}$ for $1 \mathrm{~min}$ and then cooled to room temperature slowly in buffer $\mathbf{A}(150 \mathrm{mM} \mathrm{NaCl}, 25 \mathrm{mM}$ HEPES, pH 7.6). An adenosine solution (1.5 mM) was titrated into the aptamer and the amount of released heat was measured after each addition using a MicroCal ITC instrument, allowing the dissociation constant $\left(K_{d}\right)$ to be accurately determined. At $\mathrm{pH} 7.5$, we obtained a $K_{d}$ of $13.1 \pm$ $1.8 \mu \mathrm{M}$ (Figure 2A), which was comparable to the literature reported $6 \pm 3 \mu \mathrm{M} .{ }^{27}$ At $\mathrm{pH} 5.5$, the $K_{d}$ increased slightly to $\sim 19.6 \mu \mathrm{M}$ (Figure 2B). At $\mathrm{pH} 3.5$, however, no obvious binding was observed (Figure 2C). Only when the adenosine concentration was increased to $10 \mathrm{mM}$, did we obtain a binding curve with a $K_{d}$ of $202 \mu \mathrm{M}$ (Figure 2C, inset). At $\mathrm{pH}$ 3.5, the $\mathrm{A}$ and $\mathrm{C}$ bases in DNA and the target molecule adenosine are partially protonated, which is likely to interfere with hydrogen bonding and charge interactions. The $\mathrm{pH}$ effect on a cocaine aptamer binding was also recently reported. ${ }^{28}$ This experiment supports our hypothesis that the adenosine aptamer binding is weakened at low $\mathrm{pH}$.

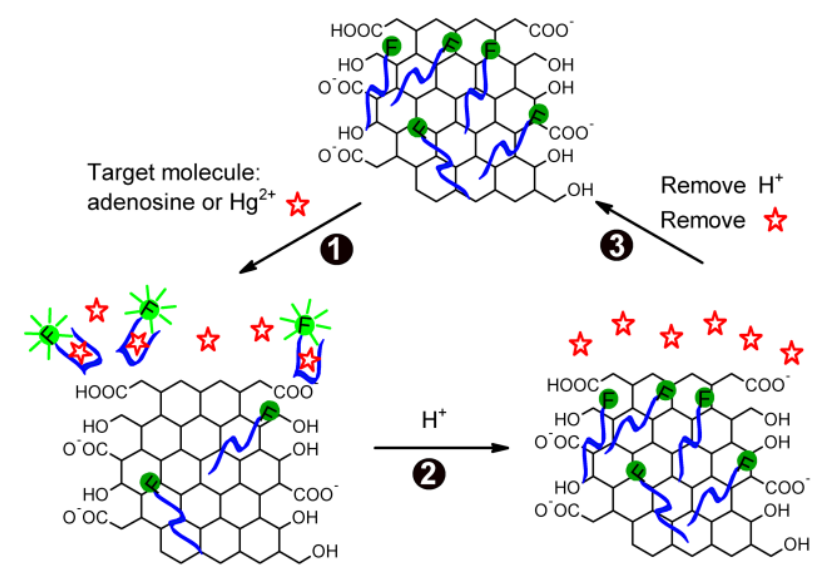

Adenosine aptamer FAM-ACCTGGGGGAGTATTGCGGAGGAAGGT-3' $\mathrm{Hg}^{2+}$ binding DNA FAM-TTCTTTCTTCCCCTTGTTTGTT-3'

Fig. 1 Schematic presentation of sensor operation (step 1) and regeneration (step 2 and 3). The aromatic rings on GO are not drawn for the clarity of the figure. The aptamer sequences are listed from the $5^{\prime}$ to $3^{\prime}$-end. FAM denotes for carboxyfluorescein.

We recently demonstrated that DNA binding to GO was stronger at lower $\mathrm{pH}^{29}$ Both DNA and GO are negatively charged and they experience a strong repulsive force. The electrostatic repulsion can be reduced by adding salt as well as by decreasing $\mathrm{pH}$. At low $\mathrm{pH}$, more surface carboxylic acid 
groups are protonated to reduce the repulsion between DNA and GO. For example, the $\xi$-potential of GO has been reported to increase by lowering the $\mathrm{pH} \cdot{ }^{30}$ Here, we also tested the $\mathrm{pH}$ dependent binding using the adenosine aptamer. GO was synthesized as described previously. ${ }^{29}$ The adenosine sensor was prepared by incubating $500 \mathrm{nM}$ FAM-labeled adenosine aptamer with $100 \mu \mathrm{g} / \mathrm{ml} \mathrm{GO}$ for $1 \mathrm{hr}$ in buffer B $(150 \mathrm{mM}$ $\mathrm{NaCl}, 1 \mathrm{mM} \mathrm{MgCl} 2,25 \mathrm{mM}$ HEPES, $\mathrm{pH}$ 7.6). The mixture was centrifuged at $15000 \mathrm{rpm}$ for $20 \mathrm{~min}$ and the GO/aptamer complex formed a pellet at the bottom. The supernatant was removed and the sensor was washed once with buffer $B$ to remove unbound aptamers. Since GO can completely quench adsorbed fluorophores, the amount of adsorption can be calculated from the fluorescence intensity in the supernatant solution. Before measurement, the supernatant $\mathrm{pH}$ was adjusted to 8.5 to ensure that the FAM fluorophore had high quantum yield. At high ionic strength (e.g. buffer A), adsorption was close to $100 \%$ at all the three $\mathrm{pH}$ values tested (Figure 2D, black bars). At a lower ionic strength (only 25 $\mathrm{mM}$ buffer, no additional salt), the aptamer showed almost no binding to $\mathrm{GO}$ at $\mathrm{pH} 7.5$ but close to quantitative binding at lower pH's (gray bars). This experiment suggests that by tuning the $\mathrm{pH}$ and ionic strength of the solution, the adsorption of DNA on GO can be controlled. Importantly, the $\mathrm{pH}$ effect was synergistic; lowering the $\mathrm{pH}$ decreased aptamer binding but promoted GO binding.

After demonstrating the effect of $\mathrm{pH}$ on bindnig, we next tested sensor regeneration. Mixing the FAM-labeled adenosine aptamer with GO resulted in low fluorescence and addition of $2 \mathrm{mM}$ adenosine induced fast fluorescence enhancement, reaching a plateau in $\sim 10 \mathrm{~min}$ (Figure $3 \mathrm{~A}$, solid black curve) ${ }^{12-14}$ This kinetic experiment was carried out in a volume of $50 \mu \mathrm{L}$ using a microplate reader (Infinite F200Pro, Tecan). The sample was subsequently acidified by incubating with $500 \mathrm{mM} \mathrm{pH} 3.5$ citrate buffer for $40 \mathrm{~min}$ for regeneration. At this $\mathrm{pH}$, the aptamer should release the bound adenosine and re-adsorb onto the GO surface. The sample was centrifuged at $15000 \mathrm{rpm}$ for $20 \mathrm{~min}$ to precipitate GO and the supernatant was removed. For comparison, another sample was regenerated in the same way but in a $\mathrm{pH} 7.5$ buffer (50mM HEPES, blue curve). After regeneration, both samples were dispersed in buffer B and adenosine was again added. Interestingly, fluorescence enhancement was observed for both samples. The final fluorescence reached about half of the original value for the sample regenerated at $\mathrm{pH} 3.5$ but less than $20 \%$ for the one washed at $\mathrm{pH}$ 7.5. The fact that the $\mathrm{pH}$ 3.5 sample showed a much higher fluorescence supported that a low $\mathrm{pH}$ was crucial for shuttling the DNA back to the GO surface. It was noted that the regeneration was incomplete even for the $\mathrm{pH} 3.5$ sample, which was attributed to the lost of GO during the centrifugation/washing steps. If the fold of fluorescence enhancement was compared, the change for the regenerated sensor was comparable to that for the freshly prepared one (Figure 3C, black bars). However, a significant drop was observed for the samples regenerated in the $\mathrm{pH} 7.5$ buffer (gray bars). The similarity in the relative fluorescence increase supports that the performance of the aptamer/GO sensor was not affected by regeneration and the decreased fluorescence intensity was due to the lost of sample during the washing steps. A second regeneration was subsequently carried out and similar results were also observed.
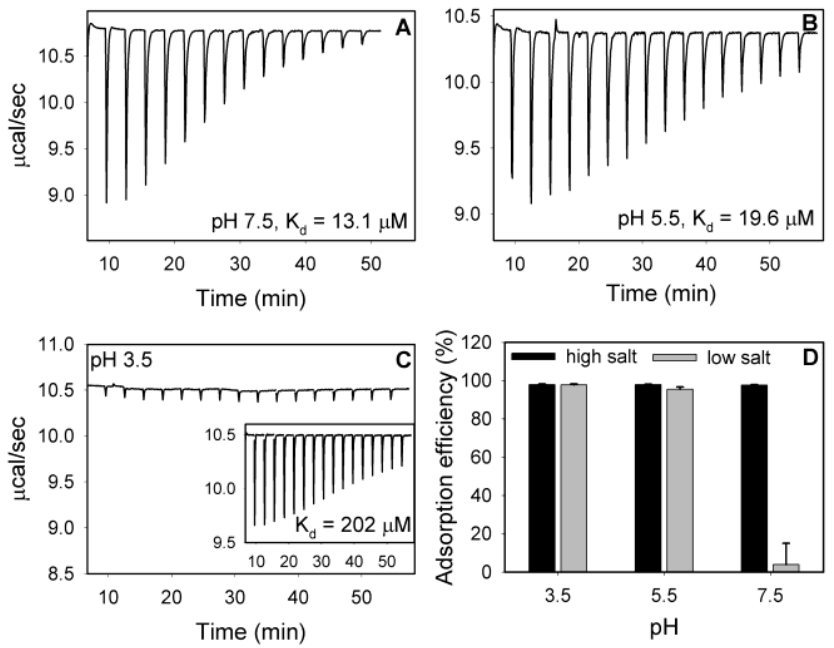

Fig. 2 ITC traces of adenosine aptamer binding at $\mathrm{pH} 7.5$ (A), 5.5 (B), and 3.5 (C). (D) Salt and pH-dependent binding of the adenosine aptamer by GO.

Interestingly, the sample washed at $\mathrm{pH} 7.5$ also showed a small increase after regeneration. After the initial addition of adenosine to the freshly prepared sensor, the fluorescence signal reached plateau before regeneration and all DNA in the supernatant was washed away at $\mathrm{pH}$ 7.5. This result suggested that not all the aptamers were desorbed from GO in the presence of $2 \mathrm{mM}$ adenosine. To confirm this, $4 \mu \mathrm{M}$ of the complementary DNA (c-DNA) of the adenosine aptamer was also used to desorb the FAM-labeled aptamer DNA. The $K_{d}$ for the c-DNA binding should be smaller than $10^{-18} \mathrm{M},{ }^{31}$ which is 12 orders of magnitude higher than that for adenosine binding. As shown in Figure 3A (black curve), the aptamer desorbed by adenosine was only about half of that desorbed by the c-DNA. This experiment confirmed the presence of residual aptamers on the GO surface after adenosine addition and the fluorescence signal after regeneration at $\mathrm{pH} 7.5$ was attributed to these residual DNA.
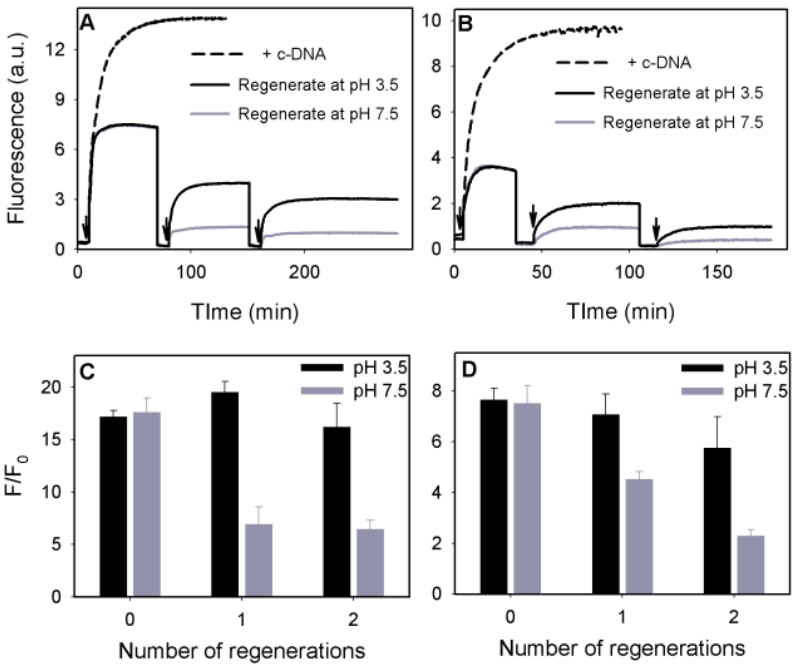
Fig. 3. Sensor regeneration. Kinetics of fluorescence change for the adenosine (A) and $\mathrm{Hg}^{2+}$ sensor (B) before and after regeneration at low or high $\mathrm{pH}$. For comparison, desorption of aptamer by the c-DNA was also tested. The target molecules were added at time points indicated by the black arrows. The fold of fluorescence enhancement (signa-to-background ratio) after adding $2 \mathrm{mM}$ adenosine (C) or $2 \mu \mathrm{M} \mathrm{Hg}^{2+}$ (D).

The dual control of the adenosine aptamer adsorption allowed the use of this system as a logic gate. To test this, four GO/aptamer samples were prepared to be at high (7.5) or low (3.5) $\mathrm{pH}$ in the presence of high $(2 \mathrm{mM})$ or low $(0 \mathrm{mM})$ adenosine. After centrifugation, the supernatant fluorescence was then measured at $\mathrm{pH} 8.5$ to avoid $\mathrm{pH}$-related FAM fluorescence artifacts. We found that high fluorescence was achieved only at high $\mathrm{pH}$ and high adenosine (Figure 4A). This system therefore acts as an AND gate (Figure 4B). This study also showed that the $\mathrm{pH}$ effect was predominate and at low $\mathrm{pH}$; no desorption occurred even in the presence of adenosine.

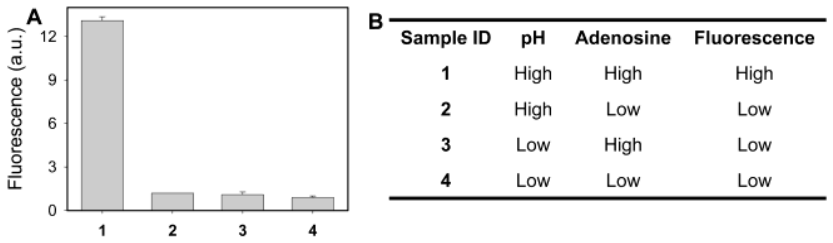

Fig. 4. (A) The supernatant fluorescence intensity of $\mathrm{GO} /$ adenosine aptamer samples in different $\mathrm{pH}$ and adenosine conditions. (B) Tabulated results in (A), where high $\mathrm{pH}=7.5$, low $\mathrm{pH}=3.5$, high adenosine $=2 \mathrm{mM}$ and low adenosine $=0$.

To test the generality of this approach, we next used a FAM-labeled mercury binding DNA. This DNA is rich in thymine (see Figure 1 for sequence) and $\mathrm{Hg}^{2+}$ can be chelated between two thymines to form the $\mathrm{T}-\mathrm{Hg}^{2+}-\mathrm{T}$ base pair. Since mercury binding to the thymine base is accompanied by the release of the imino proton, ${ }^{32,} 33$ lowering $\mathrm{pH}$ should reduce $\mathrm{Hg}^{2+}$ binding. The same regeneration experiments were performed except that the reaction buffer was changed to 150 $\mathrm{mM} \mathrm{NaNO} 3,5 \mathrm{mM}$ Tris nitrate, $\mathrm{pH}$ 8.0. Similarly, we achieved sensor regeneration by lowering the solution $\mathrm{pH}$ as shown in Figure 3B. In this case, desorption induced by $2 \mu \mathrm{M}$ $\mathrm{Hg}^{2+}$ was also incomplete and was only $\sim 40 \%$ of that by adding the c-DNA. The fold of fluorescence increase for samples regenerated at $\mathrm{pH} 3.5$ was also more consistent than those regenerated at $\mathrm{pH} 7.5$ (Figure 3D).

In summary, we have demonstrated the synergistic $\mathrm{pH}$ effect on the binding of aptamer to its target and to GO. In this system, it is possible to achieve sensor regeneration without covalent immobilization. This method should work for small molecule and metal ion targets. DNA or protein targets, however, may also bind to the GO surface with a high affinity; effective removal of such target molecules may not be achieved. This work suggests that a precise control of binding at the bio-nano interface can be achieved with a fundamental understanding of the molecular and materials properties.
This work was supported by the University of Waterloo and the Discovery Grant of NSERC of Canada. We thank Mr. Jason Da Costa and Professor Thorsten Dieckmann for assistance with the ITC experiment.

\section{Notes and references}

${ }^{a}$ Department of Chemistry, Waterloo Institute for Nanotechnology, University of Waterloo, Waterloo, Ontario, N2L 3G1, Canada. Fax: 519 7460435; Tel: 5198884567 Ext.38919; E-mail: liujw@uwaterloo.ca

1. N. L. Rosi and C. A. Mirkin, Chem. Rev., 2005, 105, 1547-1562.

2. E. Katz and I. Willner, Angew. Chem. Int. Ed., 2004, 43, 6042-6108.

3. U. Feldkamp and C. M. Niemeyer, Angew. Chem. Int. Ed., 2006, 45, 1856-1876.

4. H. Wang, R. H. Yang, L. Yang and W. H. Tan, Acs Nano, 2009, 3, 2451-2460.

5. K. S. Novoselov, A. K. Geim, S. V. Morozov, D. Jiang, Y. Zhang, S. V. Dubonos, I. V. Grigorieva and A. A. Firsov, Science, 2004, 306, 666-669.

6. A. K. Geim and K. S. Novoselov, Nat. Mater., 2007, 6, 183-191.

7. M. J. Allen, V. C. Tung and R. B. Kaner, Chem. Rev., 2009, 110, 132-145.

8. C. N. R. Rao, A. K. Sood, K. S. Subrahmanyam and A. Govindaraj, Angew. Chem. Int. Ed., 2009, 48, 7752-7777.

9. Y. Q. Wen, F. F. Xing, S. J. He, S. P. Song, L. H. Wang, Y. T. Long, D. Li and C. H. Fan, Chem. Comm., 2010, 46, 2596-2598.

10. S. J. He, B. Song, D. Li, C. F. Zhu, W. P. Qi, Y. Q. Wen, L. H. Wang, S. P. Song, H. P. Fang and C. H. Fan, Adv. Funct. Mater., 2010, 20, 453-459.

11. M. Zhang, B.-C. Yin, W. Tan and B.-C. Ye, Biosens. Bioelectron., 2011, 26, 3260-3265.

12. Y. J. Song, K. G. Qu, C. Zhao, J. S. Ren and X. G. Qu, Adv. Mater., 2010, 22, 2206-2210.

13. Y. Wang, Z. H. Li, D. H. Hu, C. T. Lin, J. H. Li and Y. H. Lin, J. Am. Chem. Soc., 2010, 132, 9274-9276.

14. C.-H. Lu, J. Li, M.-H. Lin, Y.-W. Wang, H.-H. Yang, X. Chen and G.-N. Chen, Angew. Chem. Int. Ed., 2010, 49, 8454-8457.

15. L. F. Sheng, J. T. Ren, Y. Q. Miao, J. H. Wang and E. K. Wang, Biosens. Bioelectron., 2011, 26, 3494-3499.

16. C. Liu, Z. Wang, H. Jia and Z. Li, Chem. Comm., 2011, 47, 46614663.

17. H. X. Chang, L. H. Tang, Y. Wang, J. H. Jiang and J. H. Li, Anal. Chem., 2010, 82, 2341-2346.

18. H. F. Dong, W. C. Gao, F. Yan, H. X. Ji and H. X. Ju, Anal. Chem., 2010, 82, 5511-5517.

19. X. H. Wang, C. Y. Wang, K. G. Qu, Y. J. Song, J. S. Ren, D. Miyoshi, N. Sugimoto and X. G. Qu, Adv. Funct. Mater., 2010, 20, 3967-3971.

20. H. Jang, Y. K. Kim, H. M. Kwon, W. S. Yeo, D. E. Kim and D. H. Min, Angew. Chem. Int. Ed., 2010, 49, 5703-5707.

21. C. H. Lu, H. H. Yang, C. L. Zhu, X. Chen and G. N. Chen, Angew. Chem. Int. Ed., 2009, 48, 4785-4787.

22. F. Li, Y. Huang, Q. Yang, Z. T. Zhong, D. Li, L. H. Wang, S. P. Song and C. H. Fan, Nanoscale, 2010, 2, 1021-1026.

23. F. Liu, J. Y. Choi and T. S. Seo, Biosens. Bioelectron., 2010, 25, 2361-2365. 
24. C. H. Lu, J. Li, J. J. Liu, H. H. Yang, X. Chen and G. N. Chen, Chem. Eur. J., 2010, 16, 4889-4894.

25. W. Wu, H. Hu, F. Li, L. Wang, J. Gao, J. Lu and C. Fan, Chem. Comm., 2011, 47, 1201-1203.

26. Y. Y. Shao, J. Wang, H. Wu, J. Liu, I. A. Aksay and Y. H. Lin, Electroanalysis, 2010, 22, 1027-1036.

27. D. E. Huizenga and J. W. Szostak, Biochemistry, 1995, 34, 656-665.

28. M. A. D. Neves, O. Reinstein, M. Saad and P. E. Johnson, Biophys. Chem., 2010, 153, 9-16.

29. M. Wu, R. Kempaiah, P.-J. J. Huang, V. Maheshwari and J. Liu, Langmuir, 2011, 27, 2731-2738.

30. L. J. Cote, J. Kim, Z. Zhang, C. Sun and J. X. Huang, Soft Matter, 2010, 6, 6096-6101.

31. A. K. R. Lytton-Jean and C. A. Mirkin, J. Am. Chem. Soc., 2005, 127, 12754-12755.

32. Y. Miyake, H. Togashi, M. Tashiro, H. Yamaguchi, S. Oda, M. Kudo, Y. Tanaka, Y. Kondo, R. Sawa, T. Fujimoto, T. Machinami and A. Ono, J. Am. Chem. Soc., 2006, 128, 2172.

33. A. Ono and H. Togashi, Angew. Chem. Int. Ed., 2004, 43, 4300-4302. 